\title{
Seroprevalence and Parasite Rates of Plasmodium malariae in a High Malaria Transmission Setting of Southern Nigeria
}

\author{
Eniyou C. Oriero, ${ }^{1 \star}$ Adeola Y. Olukosi, ${ }^{2}$ Olabisi A. Oduwole,${ }^{3}$ Abdoulaye Djimde, ${ }^{4}$ Umberto D’Alessandro, ${ }^{1}$ Martin M. Meremikwu, ${ }^{3}$ \\ and Alfred Amambua-Ngwa ${ }^{1}$ \\ ${ }^{1}$ Medical Research Council Unit The Gambia at LSHTM, Banjul, The Gambia; ${ }^{2}$ Nigerian Institute of Medical Research (NIMR), Lagos, Nigeria; \\ ${ }^{3}$ Calabar Institute of Tropical Disease Research and Prevention, University of Calabar Teaching Hospital, Calabar, Nigeria; ${ }^{4}$ Department \\ of Epidemiology of Parasitic Diseases, Malaria Research and Training Center, University of Science, Techniques and Technology \\ of Bamako, Bamako, Mali
}

\begin{abstract}
Although Plasmodium falciparum continues to be the main target for malaria elimination, other Plasmodium species persist in Africa. Their clinical diagnosis is uncommon, whereas rapid diagnostic tests (RDTs), the most widely used malaria diagnostic tools, are only able to distinguish between $P$. falciparum and non-falciparum species, the latter as "pan-species." Blood samples from health facilities were collected in southern Nigeria (Lagos and Calabar) in 2017 (October-December) and Calabar only in 2018 (October-November), and analyzed by several methods, namely, microscopy, quantitative real-time PCR (qPCR), and peptide serology targeting candidate antigens (Plasmodium malariae apical membrane antigen, $P$. malariae lactose dehydrogenase, and $P$. malariae circumsporozoite surface protein). Both microscopy and qPCR diagnostic approaches detected comparable proportions ( $80 \%)$ of all RDT-positive samples infected with the dominant $P$. falciparum malaria parasite. However, higher proportions of non-falciparum species were detected by qPCR than microscopy, $10 \%$ against $3 \%$ infections for $P$. malariae and $3 \%$ against $0 \%$ for Plasmodium ovale, respectively. No Plasmodium vivax infection was detected. Infection rates for $P$. malariae varied between age-groups, with the highest rates in individuals aged $>5$ years. Plasmodium malariae-specific seroprevalence rates fluctuated in those aged $<10$ years but generally reached the peak around 20 years of age for all peptides. The heterogeneity and rates of these non-falciparum species call for increased specific diagnosis and targeting by elimination strategies.
\end{abstract}

\section{INTRODUCTION}

Current global efforts toward malaria elimination target the dominant malaria parasites, Plasmodium falciparum and Plasmodium vivax. ${ }^{1}$ However, there are three other malaria species infecting humans, with Plasmodium malariae being the most common worldwide, including sub-Saharan Africa (SSA). ${ }^{2}$ Plasmodium malariae causes the so-called quartan malaria, as the onset of fever occurs in an interval of 3-4 days, because of its 72 -hour blood stage life cycle. ${ }^{3,4}$ Compared with falciparum malaria, it is a relatively benign infection, possibly due to low parasite densities, although it can cause severe anemia ${ }^{5}$ and nephrotic syndrome in both children and adults. ${ }^{6,7}$ Plasmodium malariae is often found in coinfections with other Plasmodium species, ${ }^{3}$ and because of this and the focus on $P$. falciparum, its biology, prevalence, and specific public health impact have mostly been understudied. Previous studies in areas of seasonal transmission, such as the Gambia, have reported $P$. malariae to be responsible for about half of the malaria episodes observed outside the transmission season. ${ }^{8}$ The implications of this dynamics are poorly understood in terms of sustained transmission, acquisition of antimalarial drug resistance, and malaria elimination.

Species-specific diagnosis of $P$. malariae is uncommon in routine malaria diagnosis; rapid diagnostic tests (RDTs), the most widely used diagnostic tools, predominantly target $P$. falciparum, whereas other Plasmodium species are identified

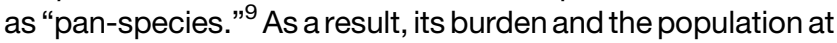
risk in most endemic areas remain largely unknown. A recent study reported persistent transmission of $P$. malariae over a 22-year period in Tanzania, suggesting that the decline in

${ }^{*}$ Address correspondence to Eniyou C. Oriero, Medical Research Council Unit The Gambia at LSHTM, Atlantic Road, Fajara, P.O. Box 273, Banjul, The Gambia. E-mail: eoriero@mrc.gm
P. falciparum prevalence could provide a favorable ecological niche for other malaria parasite species. ${ }^{10}$ This has also recently been reported for Plasmodium knowlesi, a zoonotic malaria species, in Malaysia. ${ }^{11}$

There were an estimated 228 million cases of malaria worldwide in $2018,93 \%$ in Africa, with Nigeria contributing approximately $25 \%$ of these. ${ }^{1}$ Malaria transmission in Nigeria is perennial, mostly due to conducive geographic landscape, high temperatures, and rainfall; about $85 \%$ of the population lives in areas of mesoendemic transmission. ${ }^{12}$ Although $P$. falciparum is the dominant species, responsible for more than $95 \%$ of cases of clinical malaria, P. malariae is found in $9.8 \%$ of malaria cases, mostly as mixed infection. ${ }^{12}$ Nevertheless, its prevalence, mostly determined by molecular methods, can be as high as $26 \% .{ }^{13}$ Here, we report the detection of $P$. malariae infections from two sampling surveys aimed to describe the molecular and seroprevalence of this malaria parasite species in individuals attending health facilities in two geopolitical and climatic zones of southern Nigeria.

\section{METHODS}

Study site and Plasmodium spp. survey. A first survey was carried out between October and December 2017 to compare P. malariae infections in two study sites in southern Nigeria, Lagos (lkorodu local government area) in the southwest zone and Calabar, Cross River state (Akpabuyo local government area) in the southeast zone (Figure 1A). Suspected malaria cases sent for malaria test at the study health facilities were screened using a pan-species RDT kit, SD Bioline, Cat No 05FK60 (Abbott Diagnostics, Lake Forest, IL), after providing written informed consent to participate in the study. Blood sample was then collected from RDT-positive individuals for thick blood film preparation for microscopy and dried blood spots (DBSs) for molecular detection of 
A

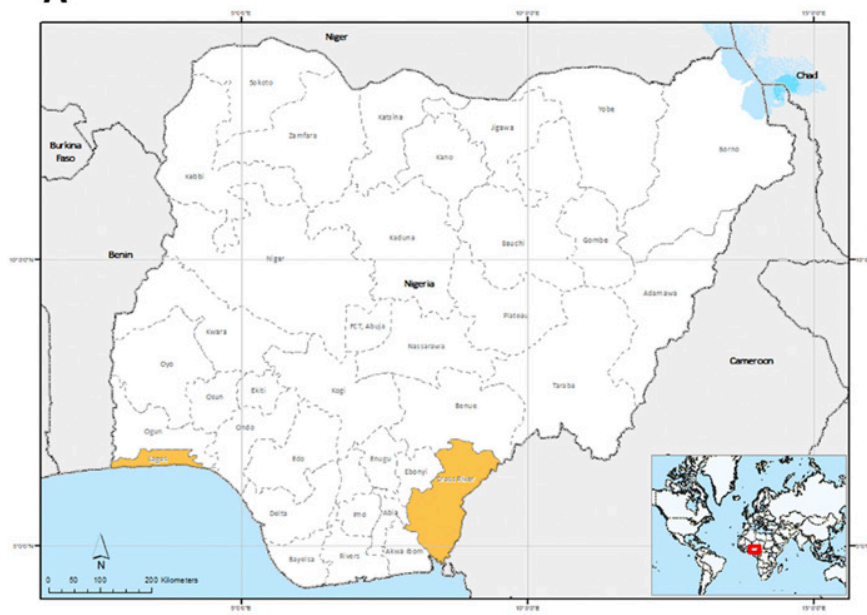

B

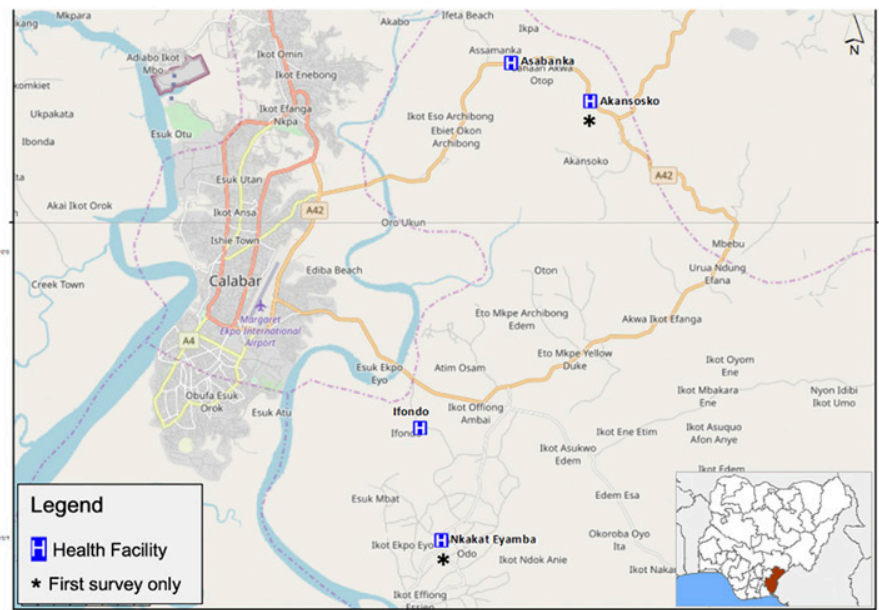

FIGURE 1. Map of study sites in Nigeria showing (A) Lagos (southwest zone) and Cross River (south-south zone) states; and (B) health facilities in Cross River state, where participants were recruited. This figure appears in color at www.ajtmh.org.

Plasmodium species and serology assays. At the peak of the following malaria transmission season (November/December 2018), a second survey was carried out in Calabar only because of the higher prevalence of $P$. malariae, expanding to additional health facilities to increase catchment, and the total numbers screened within the sample collection period (Figure 1B). Following prior community sensitization, participants who reported at the study health facilities with suspected malaria were recruited, and samples were collected from consenting individuals as described in the first survey, irrespective of their RDT results.

Lagos is the most populous city in Africa, with a population of approximately 15 million people; it has a tropical savanna climate, with a wet season between April and October, and a dry season in the following months. The wettest month is June, with mean precipitation of $315.5 \mathrm{~mm}$ (12.42 in). Located near the equator, Lagos has minimal seasonal temperature variation, with high temperatures ranging between $28.3^{\circ} \mathrm{C}$ and $32.9^{\circ} \mathrm{C} .{ }^{14}$ Calabar has a tropical monsoon climate, with a long wet season (February-November) and a short dry season covering the remaining 2 months. Temperatures are relatively constant throughout the year, with average high temperatures ranging between $25^{\circ} \mathrm{C}$ and $28^{\circ} \mathrm{C}$; relative humidity is high with most months of the year, recording a monthly mean value of $80 \%$, and annual average precipitation more than $3,000 \mathrm{~mm}$ (120 in). ${ }^{15,16}$

Ethical and sample size considerations. Ethical approval was obtained from the respective authorities in Lagos and Cross River state, references: IRB/17/038, CRSMOH/RP/ $\mathrm{REC} / 2017 / 545$, and $\mathrm{CRSMOH} / \mathrm{RP} / \mathrm{REC} / 2017 / 809$. Written informed consent was obtained for adult participants, whereas parental or guardian consent was obtained for minors. Participants did not receive any compensation for their participation in study. All individuals diagnosed with malaria by RDTs were treated accordingly, whereas febrile individuals with a negative RDT result were referred to the health facility staff for further investigation. A minimum of 500 samples per site was required to be screened in each survey, assuming maximum malaria prevalence of $50 \%$ in suspected cases with $95 \% \mathrm{Cl}$ and $5 \%$ margin of error and an additional $30 \%$ adjustment for possible incomplete data by any of the detection methods.

Detection of Plasmodium spp. parasites. A pan-species RDT kit (SD Bioline, Cat No 05FK60), which qualitatively and differentially detects histidine-rich protein II antigen of $P$. falciparum and lactate dehydrogenase (pLDH) of Plasmodium species in human whole blood, was used for malaria screening according to the manufacturers' protocol. Any sample with either P.f, pan, or both lines in addition to the control line was considered positive. Thick blood microscopy slides stained with $10 \%$ Giemsa solution were examined across 100 highpower fields by two microscopists and parasite counts recorded per number of white blood cells (WBCs) observed. In case of $\geq 20 \%$ variance in counts between primary and secondary microscopists, the counts closest to that of a third reader were retained. A slide was declared negative only after observing no parasite in 100 microscopic high-power fields, whereas parasite density was calculated using WHO protocols; the number of parasitized erythrocytes against 8,000 WBCs ${ }^{17}$ was expressed as parasitaemia/mL of blood.

For molecular diagnosis of Plasmodium spp., DNA was extracted from DBS samples using the QIAamp DNA Mini Kit (Qiagen, Hilden, Germany) according to manufacturers' protocols. For the preliminary survey, the presence of Plasmodium species was detected using a commercial species-specific quantitative real-time PCR (qPCR) assay, Genesig ${ }^{\circledR}$ Plasmodium species kits (Primerdesign ${ }^{\mathrm{TM}}$ Ltd., Chandler's Ford, United Kingdom) according to manufacturers' protocol. Subsequently, the presence of $P$. malariae and Plasmodium ovale was detected using a custom qPCR assay targeting the Plasmepsin gene, ${ }^{18}$ which showed comparable results with the Genesig kit (Primerdesign Ltd.), whereas the presence of $P$. vivax was not assessed further. Pre-amplification was performed using $0.1 \mu \mathrm{M}$ of the forward and reverse primers for $P$. malariae (CCWR2K1_Fwd TTCAGTCAGGATATGTAAAACAAAATTATTTAGGTA; CCWR2 K1_Rev CCTACTTCCCCTTCACCATAAAACA) and $P$. ovale (CCRR9V5_Fwd ACTCTTGGTTATTTGTCTGCACCTT; CCRR9V5_ Rev CTATGTTACCATAAACAGGTTCTAAATCATCTGT), $1 \times$ Qiagen Multiplex Master Mix (QIAGEN) and $5 \mu \mathrm{L}$ of template DNA in a $15-\mu \mathrm{L}$ reaction. The main amplification reaction contained 
$3 \mu \mathrm{L}$ of pre-amplified products in a $15-\mu \mathrm{L}$ total reaction volume and was performed using $1 \times$ qPCR Taqman Universal Master Mix (Thermo Fischer Scientific, Waltham, MA), $0.4 \mu \mathrm{M}$ of the forward and reverse primers, and $0.2 \mu \mathrm{M}$ of the labeled probe ( $P$. malariae, CCWR2K1_Probe TCGTCTAGTTCTATTACGTCATTTC and $P$. ovale, CCRR9V5_Probe TCAGTTGCTTCAACAAATTT). Pre-amplification was performed under the following conditions: initial denaturation for 5 minutes at $95^{\circ} \mathrm{C}, 12$ cycles of denaturation for 30 seconds at $95^{\circ} \mathrm{C}$, annealing at $60^{\circ} \mathrm{C}$ for 1 minute, and extension for 90 seconds at $72^{\circ} \mathrm{C}$, whereas main amplification and detection were performed under the following conditions: initial denaturation for 10 minutes at $95^{\circ} \mathrm{C}, 40$ cycles of denaturation for 10 seconds at $95^{\circ} \mathrm{C}$, and data collection for 1 minute at $60^{\circ} \mathrm{C}$. Plasmodium falciparum was detected using a highly sensitive qPCR assay (varATS qPCR) targeting multi-copy subtelomeric sequences. ${ }^{19}$ Amplification reaction was performed using $1 \times$ qPCR Taqman Universal Master Mix (Thermo Fischer Scientific).

Peptide design and serology assays. Plasmodium malariae-specific peptides were designed from highly immunogenic epitopes of three $P$. malariae antigens-apical membrane antigen (AMA1), circumsporozoite surface protein (CSP), and lactose dehydrogenase (LDH) (Table 1). A P. falciparumspecific peptide was also designed from the same region as one of the P. malariae antigens - AMA1 (PmAMA1) peptides to explore species specificity. A web tool, SVMTriP (System Biology Laboratory of Chi Zhang, University of Nebraska, Lincoln, $\mathrm{NE}),{ }^{20}$ was used to predict linear antigenic epitopes from the whole $P$. malariae antigen sequences, and the peptides were queried for species specificity using NCBI BLASTP 2.9.0+ (Bethesda, MD). The regions of immunogenic epitopes containing the peptides were then compared with orthologs of predicted $P$. falciparum epitopes available on the Plasmodium database $^{21}$ and the Immune Epitope Database and Resource Analysis. ${ }^{22}$ The peptides indicating high species specificity and high immunogenicity were chosen for synthesis (thinkpeptides ${ }^{\circledR}$ Prolmmune Ltd., Oxford, United Kingdom) at 95\% purity and 1-4 mg synthesis scale.

Antibodies against the Plasmodium spp. peptides were detected by indirect ELISA on eluted filter paper samples. One disc $(6 \mathrm{~mm})$ per sample was punched and placed at the bottom of 96-well plates-flat bottom with low evaporation lids (Corning Inc., New York, NY). Serum from DBS was eluted following overnight (18 hours) incubation at $4^{\circ} \mathrm{C}$ in $150 \mu \mathrm{L}$ of reconstitution buffer (1x phosphate buffer saline; $0.2 \%$ Tween20). The reconstituted solution was equivalent to a 1: 100 dilution of whole blood, corresponding to 1:200 dilution of plasma or serum antibodies, if the blood were at $50 \%$ hematocrit. ${ }^{23}$ Peptides were coated on 96-well Nunc MaxiSorp ${ }^{\mathrm{TM}}$ Flat-Bottom Plates (Thermo Fischer Scientific) at a concentration of $3 \mu \mathrm{g} / \mathrm{mL}$. Following blocking of all remaining binding surfaces with $1 \%$ skimmed milk, DBS eluate was added at a final concentration of 1 : 2,000 with respect to the corresponding plasma sample and analyzed in duplicate wells per sample. Each ELISA plate included a positive control, which was a pool of DBS eluate from samples confirmed to be $P$. malariae positive by microscopy and qPCR. Also included in each plate were plate blanks (no serum) and negative control, which was unexposed European pooled serum. ${ }^{24}$ Secondary detection was with antibody peroxidase conjugated human IgG (H\&L) (Sigma-Aldrich, Taufkirchen, Germany), developed with Pierce ${ }^{\mathrm{TM}}$ TMB Substrate Kit (Thermo Fischer Scientific). The reaction was stopped using $50 \mu \mathrm{L}$ of Stop Solution for TMB substrates (Thermo Fischer Scientific). Optical densities (ODs) were read on the EMax ${ }^{\circledR}$ Plus microplate reader (Molecular Devices, San Jose, CA) at $450 \mathrm{~nm}$.

Data analysis. Data analysis was conducted using Microsoft Excel spreadsheet, GraphPad Prism version 8.4.2 (San Diego, CA), and Stata IC 16 (Stata Corp., College Station, TX). The prevalence of $P$. malariae and non-falciparum species detected by the different detection methods was compared from the different study sites. Analytical procedures for reducing between-plate variation in the serology included calculating the differences between the mean ODs averaged across the duplicates of the pooled positive control for each plate and the overall mean OD values across all plates, and obtaining the estimated plate effect for a specific plate by averaging the calculated differences across all pooled controls. Test samples on a given plate were then adjusted by subtracting the estimated plate effect from their OD values. ${ }^{25}$ Positivity for antimalarial peptide response was obtained by modeling their normalized fold over the negative control (FOC) values ( $\log _{2}$ transformed), using finite mixture models, and Gaussian distribution with two components. ${ }^{23,24}$ The estimated mean of the narrower distribution was used as the mean for negative samples. The cutoff for seropositivity was obtained by taking the difference between the peptide FOC values and the mean of the narrower distribution plus 2 SDs. Seroprevalence rates were analyzed according to the following age-groups: 10-20 years, 20-30 years, and $>30$ years, which corresponded with estimated the duration of exposure to malaria. A three exponent nonlinear regression model and line of best fit, using the quadratic estimation, was used to obtain the predicted probability of being seropositive for a particular peptide.

\section{RESULTS}

Summary of participants recruited. In 2017, a total of 243 $(21.7 \%)$ individuals with a positive malaria RDT result were recruited of 1,115 participants screened-520 screened from Lagos and 595 from Calabar. Rapid diagnostic test positivity rate in Lagos was $13.7 \%$ and $28.9 \%$ in Calabar (Figure 2A).

TABLE 1

List of Plasmodium spp. peptides for the serology assays

\begin{tabular}{|c|c|c|c|c|c|}
\hline $\mathrm{S} / \mathrm{N}$ & Peptide name & Peptide sequence & Sequence location & Antigen & Parasite spp. \\
\hline 1 & PmAMA1-1 & VLRKRYEEHADLMNLNDLSL & $141-160$ & AMA1 & P. malariae \\
\hline 2 & PmAMA1-2 & VKLYSLDGEKIVLPRIFISN & $405-424$ & AMA1 & P. malariae \\
\hline 3 & PmCSP & AGNAAGNAAGNAAGNAAGNE & $256-275$ & CSP & P. malariae \\
\hline 4 & PmLDH-1 & ALIVAAHGNKMVPLKRYITV & $176-169$ & LDH & P. malariae \\
\hline 5 & PmLDH-2 & LGGVLDTSRLKYYISQKLNV & $150-169$ & LDH & P. malariae \\
\hline 6 & PfAMA1 & TLDEMRHFYKDNKYVKNLDE & $194-213$ & AMA1 & P. falciparum \\
\hline
\end{tabular}


A
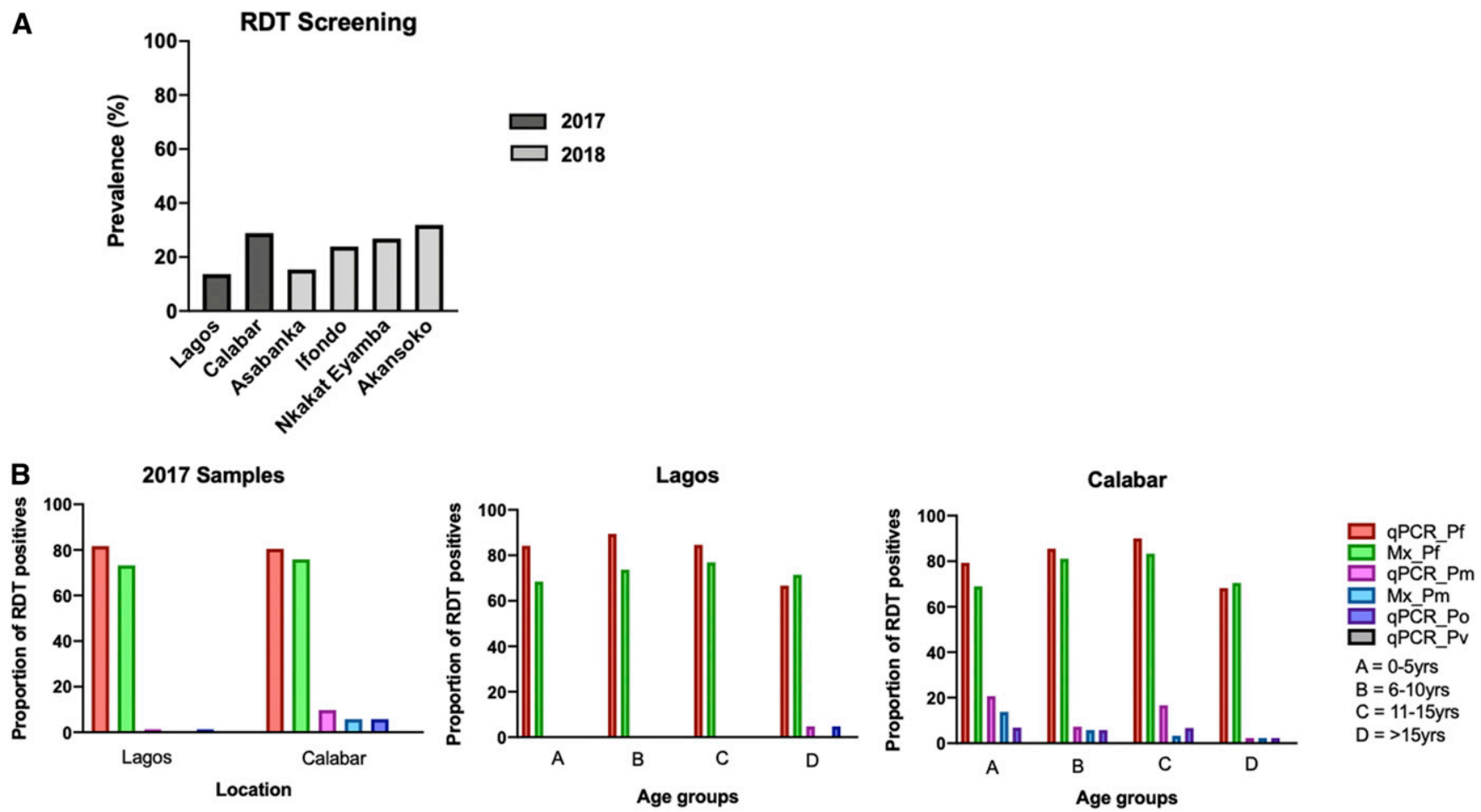

FIGURE 2. Summary of diagnostic results. (A) Prevalence of malaria in both surveys by RDTs. (B) Proportion of Plasmodium species detected by qPCR and microscopy in RDT-positive samples from the first survey. $\mathrm{Mx} \_\mathrm{Pf}=P$. falciparum detected by microscopy; $\mathrm{Mx} \_\mathrm{Pm}=P$. malariae detected by microscopy; qPCR = quantitative real-time PCR; qPCR Pf = P. falciparum detected by qPCR; qPCR Pm = P. malariae detected by qPCR; qPCR_Po = $P$. ovale detected by qPCR; qPCR_Pv $=P$. vivax detected by $\mathrm{qPCR}$. RDT = rapid diagnostic test. This figure appears in color at www.ajtmh.org.

Most of the RDT-positive cases were in Calabar (71\%, 172/ 243); children aged $6-10$ years were the largest age-group (36\%, 88/243; Table 2). The following year, a total of 798 participants were recruited from four health facilities in Calabar $(77 \%, 613 / 798)$, mostly from two of the facilities (Table 2 ). The age distribution of the participants was different from that of the preliminary survey performed in 2017; adolescents and adults represented about half of the participants $(51 \%, 410 / 798)$, followed by children aged $11-15$ years $(21 \%, 168 / 798$; Table 2$)$.

Molecular prevalence of Plasmodium species. In 2017, when only RDT-positive individuals were recruited, the proportion of Plasmodium species detected in Lagos by qPCR was $81.7 \%, 1.4 \%$, and $1.4 \%$ for $P$. falciparum, $P$. malariae, and $P$. ovale, respectively. In Calabar, the proportions detected were $80.8 \%, 9.9 \%$, and $5.8 \%$, respectively (Figure 2B). No $P$. vivax infection was detected in both locations. Similar proportions $(81.7 \%$ versus $73.2 \%$ [Lagos] and $80.5 \%$ versus $75.9 \%$ [Calabar]) of $P$. falciparum infections were detected by
qPCR and microscopy in the RDT-positive samples from the different study areas. However, more P. malariae and $P$. ovale infections were detected by qPCR, with higher proportions in Calabar than in Lagos. This emphasized the low specificity of species-specific identification of malaria parasites by microscopy. Table 3 summarizes the prevalence of Plasmodium spp. detected as mixed or mono infections in the four health facilities in Calabar. On average, relatively more non-falciparum species infections were detected in malaria samples recruited from the two facilities situated to the north of Akpabuyo local government area $(26.5 \%)$ than those to the south (18.7\%); however, this was not statistically significant $(P=0.062)$ (Figure 3). Despite observing a higher proportion of $P$. malariae infections in older individuals ( $>15$ years), the odds of having $P$. malariae as a mono-infection were highest in the $<5$ year age-group. The least odds for mono-infections were in those of ages 11-15 years (Table 4); the odds of $P$. malariae mono infections were highest in Asabanka, with

TABLE 2

Summary of participants recruited in the two malaria transmission seasons

\begin{tabular}{|c|c|c|c|c|c|c|c|c|}
\hline & \multicolumn{3}{|c|}{ First survey, $n(\%)$} & \multicolumn{5}{|c|}{ Second survey, $n$ (\%) } \\
\hline & Lagos & Calabar & Total & Akansoko HF & Asabanka HF & Ifondo $\mathrm{HF}$ & Nkakat Eyamba HF & Total \\
\hline Total & 71 & 172 & 243 & 97 & 266 & 88 & 347 & 798 \\
\hline \multicolumn{9}{|l|}{ Gender } \\
\hline Male & $31(43.7)$ & $88(51.2)$ & 119 (48.9) & 45 (46.4) & $120(45.1)$ & 38 (43.2) & $147(42.4)$ & 350 (43.9) \\
\hline Female & $40(56.3)$ & $84(48.8)$ & 124 (51.0) & 52 (53.6) & 146 (54.8) & 50 (56.8) & 200 (57.6) & 448 (56.1) \\
\hline \multicolumn{9}{|c|}{ Age-group (years) } \\
\hline $0-5$ & $19(26.7)$ & $29(16.8)$ & $48(19.7)$ & $10(10.3)$ & 49 (18.4) & $18(20.5)$ & $18(5.2)$ & 95 (11.9) \\
\hline $6-10$ & 19 (26.7) & $69(40.1)$ & 88 (36.2) & $6(6.2)$ & 71 (26.7) & $9(10.2)$ & 39 (11.2) & 125 (15.6) \\
\hline $11-15$ & 13 (18.3) & 30 (17.4) & 43 (17.6) & $7(7.2)$ & 38 (14.3) & $6(6.8)$ & 117 (33.7) & $168(21.1)$ \\
\hline$>15$ & $21(29.5)$ & $44(25.5)$ & $65(26.7)$ & 74 (76.3) & $108(40.6)$ & 55 (62.5) & $173(49.8)$ & 410 (51.4) \\
\hline
\end{tabular}


TABLE 3

Summary of non-falciparum species detected in south-south Nigeria as mixed and mono-infections by the different diagnostic methods-RDT, microscopy, and qPCR

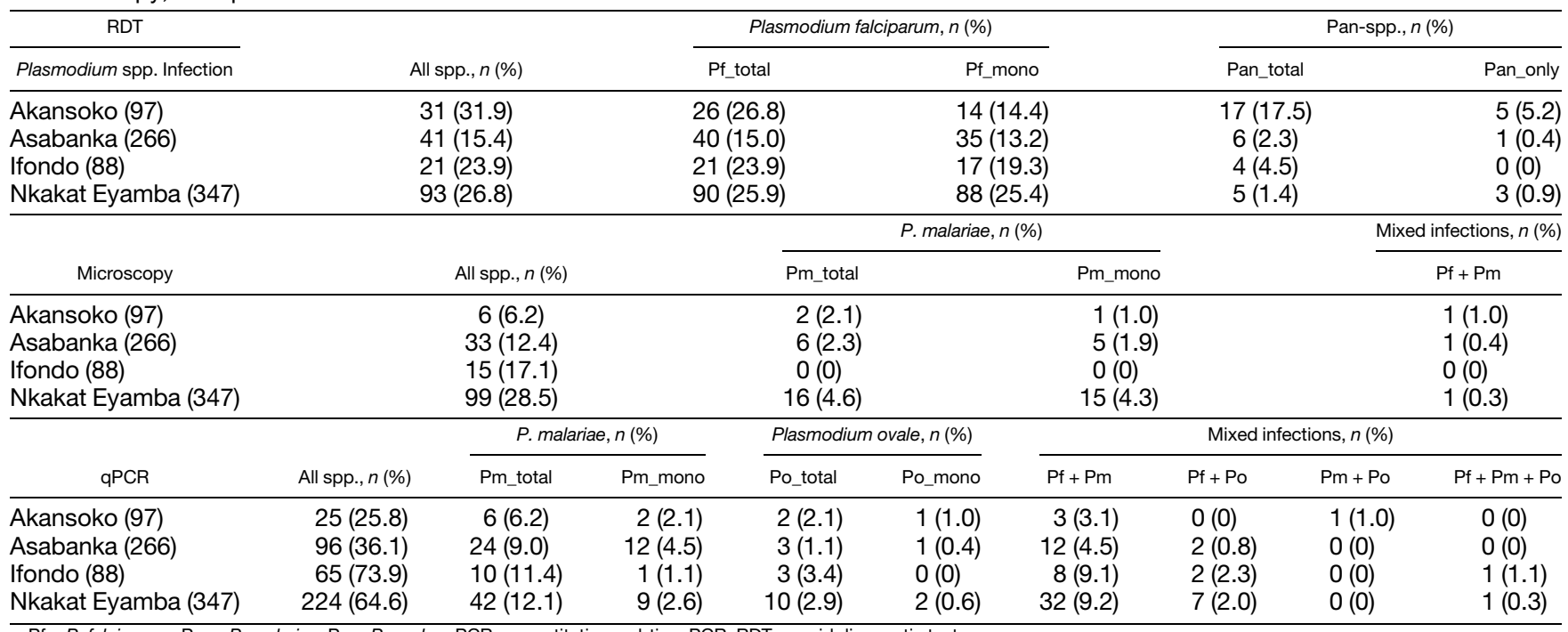

$\mathrm{Pf}=P$. falciparum; $\mathrm{Pm}=$ P. malariae; $\mathrm{Po}=P$. ovale; $\mathrm{qPCR}$ = quantitative real-time $\mathrm{PCR} ; \mathrm{RDT}=$ rapid diagnostic test.

$4.5 \%$ mono-infection, and the least in Ifondo, with $1.1 \%$ mono-infection (Tables 3 and 4).

Serological responses to the different $P$. malariae peptides. Anti-peptide lgG antibody levels were detected against each of the Plasmodium spp. peptides designed, and these were relatively higher than the median values of a pool of nonimmune sera, reported as FOC. More than $70 \%$ of the samples showed antibody reactivity levels

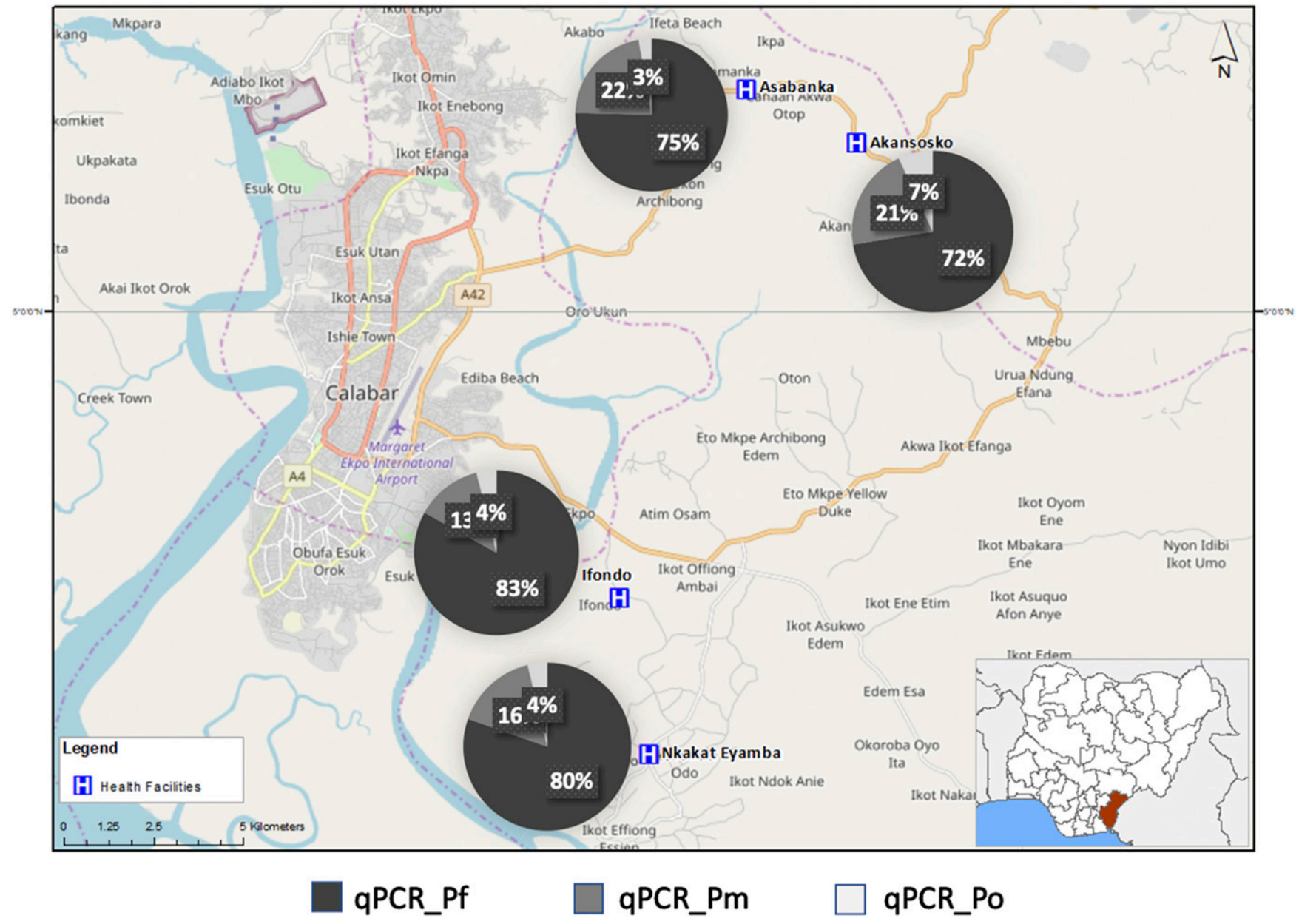

FIGURE 3. Proportion of Plasmodium species detected in the four villages in southeastern Nigeria, detected by quantitative real-time PCR (qPCR) (qPCR_Pf $=P$. falciparum; qPCR_Pm $=P$. malariae; qPCR_Po $=P$. ovale). This figure appears in color at www.ajtmh.org. 
TABLE 4

Odds of $P$. malariae occurring as mono-infections or mixed infections with other Plasmodium spp. in the four different villages and across age-groups

\begin{tabular}{|c|c|c|c|c|c|c|c|}
\hline & Mono infections & Mixed infections & Total & Odds ${ }^{*}$ & $95 \% \mathrm{Cl}$ & Odds ratio $†$ & $95 \% \mathrm{Cl}$ \\
\hline \multicolumn{8}{|l|}{ Health facility } \\
\hline Akansoko & 2 & 4 & 6 & 0.5 & $0.09-2.73$ & 4.5 & $0.25-80.57$ \\
\hline Asabanka & 12 & 12 & 24 & 1.0 & $0.45-2.23$ & 9 & $0.79-101.81$ \\
\hline Ifondo & 1 & 9 & 10 & 0.11 & $0.01-0.88$ & 1 & - \\
\hline Nkakat Eyamba & 9 & 33 & 42 & 0.27 & $0.13-0.57$ & 2.46 & $0.26-22.82$ \\
\hline \multicolumn{8}{|l|}{ Age-group (years) } \\
\hline $0-5$ & 4 & 3 & 7 & 1.33 & $0.29-5.96$ & 16.67 & $1.39-199.42$ \\
\hline $6-10$ & 3 & 9 & 12 & 0.33 & $0.09-1.23$ & 4.17 & $0.55-31.75$ \\
\hline $11-15$ & 2 & 25 & 27 & 0.08 & $0.02-0.34$ & 1 & - \\
\hline$>15$ & 15 & 21 & 36 & 0.71 & $0.37-1.39$ & 8.93 & $1.58-50.32$ \\
\hline
\end{tabular}

* Odds of $P$. malariae observed as a mono-infection compared with mixed infections.

† Odds ratio calculated relative to the least odds.

greater than the negative control for each of the peptides (PmAMA1-1 $=73.1 \%$, PmAMA1-2 = 86.7\%, P. malariae CSP $[\mathrm{PmCSP}]=78.2 \%, P$. malariae LDH $[\mathrm{PmLDH}-1]=$ $80.9 \%$, PmLDH-2 = 87.2\%, and PfAMA1 = 80.3\%). From the area under the receiver operator curve for the different peptides, the FOC responses of all the peptides could predict up to $70 \%$ of the results obtained by microscopy (range $=62.6-70.5 \%)$ and RDTs (range $=66.4-71.9 \%)$ (Figure 4). The P. malariae-specific anti-peptide antibody reactivities relative to the $P$. falciparum peptide for samples from the first survey expressed significantly higher $P$. malariae responses in Calabar than in Lagos with the exception of the PmCSP peptide, to which Lagos samples were more reactive (Supplemental Figure 1). Because of the small number of samples from Lagos and some of the health facilities in Calabar, all samples were subsequently analyzed together, and the geographical location was not considered. As observed with $P$. falciparum, seroprevalence increased with age for all the $P$. malariae peptides from the age of 10 years (Figure 5). Proportions of seropositivity in younger than 10-year age-groups were variable between antigens, with no consistent pattern between peptides (Supplemental Figure 2). The proportion of seropositivity decreased for PmLDH-1, whereas it increased for PfAMA1 for this younger agegroup.

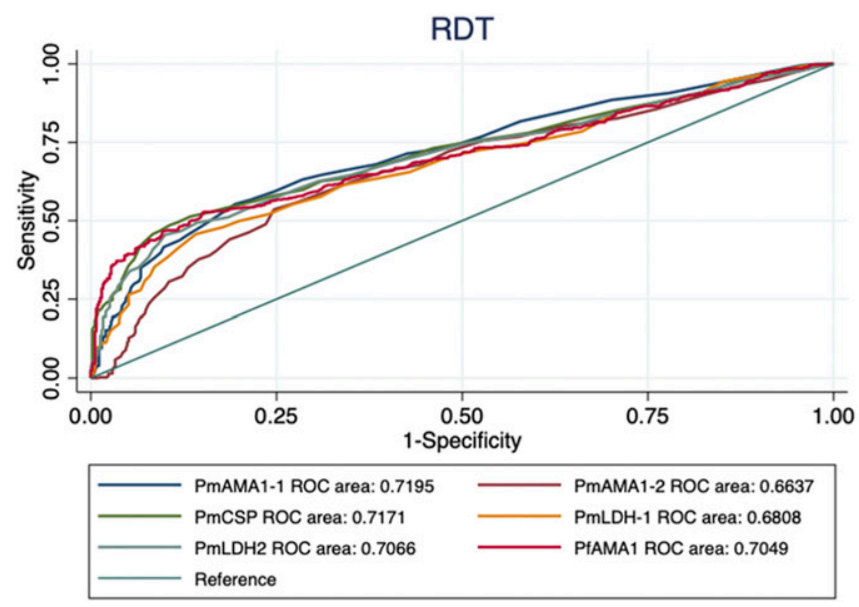

\section{DISCUSSION}

The prevalence of non-falciparum species was relatively heterogeneous in health facilities in the southwest and southsouth zones of Nigeria, supporting the need for broadening the focus of the National Malaria Control Program beyond $P$. falciparum. ${ }^{26-29}$ Nigeria accounts for an estimated $25 \%$ of global malaria cases, mostly considered to be due to $P$. falciparum infection. ${ }^{30}$ With the push for malaria eradication, this indicates that other human Plasmodium species should also be considered in current elimination strategies and evaluation of intervention outcomes. Unlike $P$. falciparum, $P$. malariae infections were more prevalent in individuals older than 5 years. Current malaria intervention strategies such as seasonal malaria chemoprevention (SMC) usually target children aged 3-59 months. Hence, older age-groups in $P$. malariae-endemic areas could serve as a reservoir for future expansion of this parasite if $P$. falciparum is selectively eliminated. Higher prevalence of $P$. malariae infections was also observed in older age-groups in Papua New Guinea based on a sensitive molecular diagnostic method, post-PCR ligase detection reaction-fluorescent microsphere assay, compared with light microscopy. ${ }^{31} \mathrm{Al}-$ though overall malaria parasite species differ, the rates for $P$. malariae infections were comparable to those reported for Papua, despite the wide geographic distance and

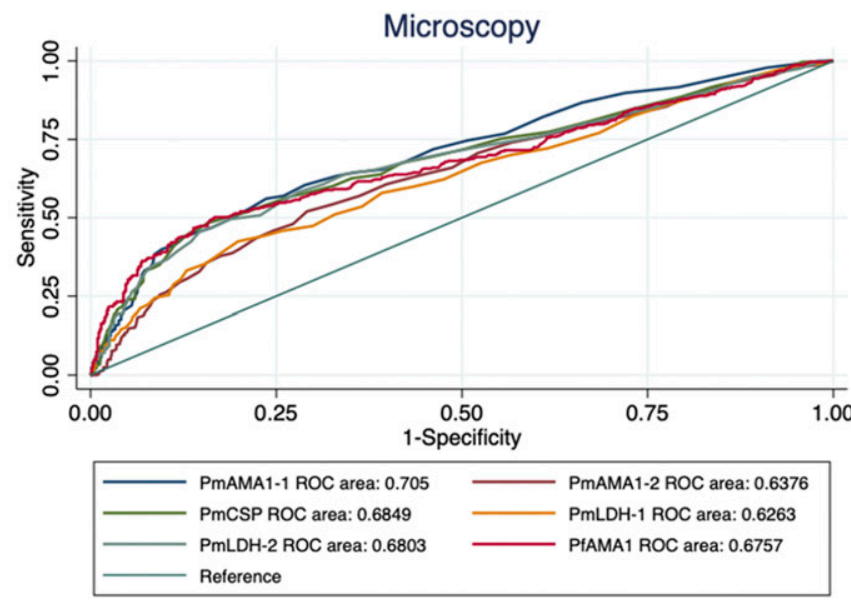

FIGURE 4. Area under the receiver operator curve $(\mathrm{ROC})$ showing comparison of fold over the negative control for each of the peptides with rapid diagnostic test (RDT) and microscopy results. This figure appears in color at www.ajtmh.org. 

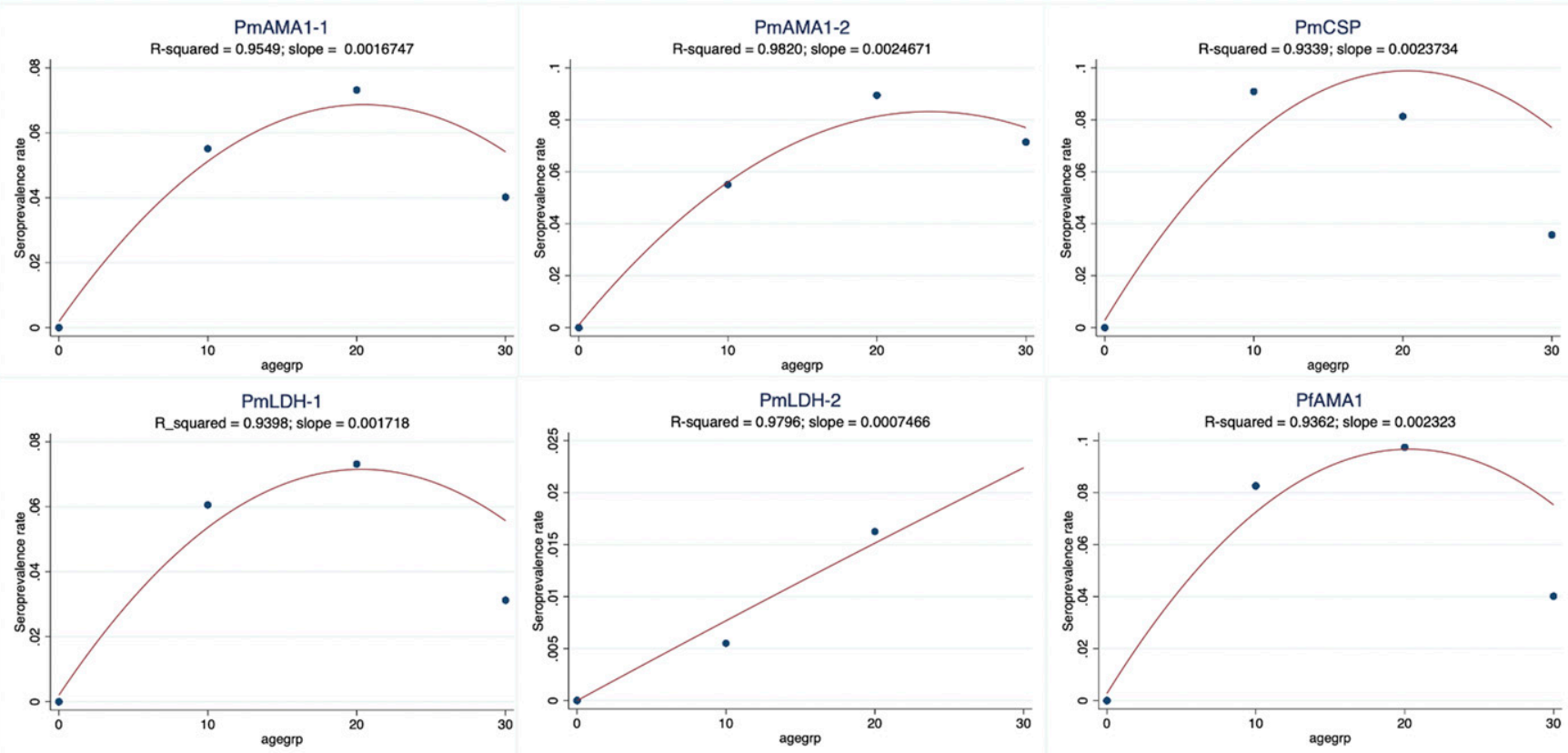

FIGURE 5. Seroprevalence rates of the different species-specific peptides in different age-groups; the slope is the estimated change of rate over age-group. This figure appears in color at www.ajtmh.org.

additional prevalence of $P$. vivax in Papua. A wider surveillance and reporting of non-falciparum malaria parasite species and broadening of future malaria elimination strategies to include all population age-groups at risk of infection with $P$. falciparum and non-falciparum species is suggested. Identification of five cases of $P$. vivax and a $10.9 \%$ prevalence of $P$. malariae in a recent study of southern Nigerian communities further corroborates our findings and need for enlarged surveys. ${ }^{29}$

Despite the lower prevalence of infection in children $<5$ years of age, the odds of $P$. malariae mono-infections were higher in this age-group than in individuals aged $>15$ years. This could reflect the level of exposure and immunity, as younger children may be protected by maternal antibodies as well as interventions such as insecticide-treated bed nets and SMC. ${ }^{32}$ The age distribution also mirrors the pattern seen for $P$. falciparum, where an age shift in high infection rates to older children has been reported across Africa following large scaleup of malaria interventions. ${ }^{33}$ The odds of $P$. malariae monoinfection also varied between health facilities, with lowest infection odds in Ifondo, which had, by molecular methods, the highest prevalence of $P$. falciparum infections. This, however, would require further investigation because of the small numbers reported here, reflected in the wide Cls. Nonetheless, some studies have reported higher $P$. malariae prevalence rates when outside the $P$. falciparum peak transmission, suggesting an interaction between the occurrence of these species. ${ }^{34-36}$ Such dynamics could be driven by co-immune mechanisms as the genomes and proteins of these species are highly similar, ${ }^{37}$ hypothetically allowing for high immune responses against $P$. falciparum to eliminate $P$. malariae from coinfections. There could also be an ecological dynamics in which higher multiplication rates and shorter cycle time of $P$. falciparum would displace $P$. malariae, which often occurs at low parasite densities and prefer to invade older red blood cells (RBCs). ${ }^{4}$ Infected RBCs are cleared by the spleen during malaria infections, ${ }^{38,39}$ which may in turn remove $P$. malariae from peripheral circulation. However, $P$. malariae has been reported to persist following treatment of mixed species malaria with artemisinin combination therapies, ${ }^{40-42}$ an indication that further studies are needed to elucidate the dynamics and consequences of malaria parasite coinfections. This will require specific surveillance tools, including sensitive and specific diagnostic and serological assays.

In the absence of whole antigen ELISA assays for $P$. malariae and other non-falciparum infections, speciesspecific peptide ELISA assays provided an alternative tool for determining seroprevalence of antibodies against $P$. malariae. Predicted peptide epitopes from $P$. malariae AMA1, CSP, and $\mathrm{LDH}$ proteins were all recognized by human IgG antibodies, evidence of specific immune responses in exposed communities. Higher relative antibodies were seen in the south-south zone with higher malaria transmission. Thus $P$. malariae elicits specific antibody responses, which could be targeted for the development of sensitive surveillance tools. Seroprevalence has been used as a surrogate for exposure, enabling the determination of force of infection and age-specific immunity for $P$. falciparum and $P$. vivax, in endemic regions. ${ }^{24,43-45}$ ELISA techniques for the detection of antibodies have also been widely used for the diagnosis of infectious diseases and have been used in malaria since the 1970 s. ${ }^{46}$ Adequately powered studies to compare and predict transmission intensity of $P$. malariae and other non-falciparum species across SSA would be useful for strategizing toward control and elimination of all malaria parasite species.

The first survey carried out in 2017 showed a lower prevalence of non-falciparum species in Lagos than in Calabar. This was not surprising as urbanization in Lagos metropolitan city, with better housing and access to health services, will limit malaria transmission despite similar vector populations. ${ }^{47} \mathrm{~A}$ consistent pattern of increased urbanization coincident with decreasing malaria transmission and elimination over the past 
century has been reported. ${ }^{48}$ Although this study was not designed to determine the epidemiology of $P$. malariae, a properly designed epidemiological study would be more informative. The non-falciparum infections in the urban area were submicroscopic and could only be detected by sensitive molecular methods. This emphasizes the challenges to malaria diagnosis with decreased prevalence and more submicroscopic infections. Sensitive and accessible diagnostic tools for routine detection of all human Plasmodium species therefore remain important. Microscopy, which is still considered the gold standard for malaria diagnosis, is laborious and time consuming for Plasmodium speciation, requiring expert microscopists. Current antigen-based pan-species RDTs also present peculiar challenges, including low sensitivity and lack of species specificity. ${ }^{49}$ The poor sensitivity for pan-species Plasmodium detection was recently demonstrated in an experimental human blood-stage model for $P$. malariae infection, where RDTs targeting pan-genus LDH enzyme $(\mathrm{pLDH})$ remained negative despite the presence of symptoms consistent with malaria 72-hour before testing. ${ }^{50}$

Most of the malaria infections detected by PCR were lowgrade infections or submicroscopic, which is a well-documented limitation of microscopy, and this may explain the high discrepancy between Plasmodium species detected by microscopy compared with PCR. As for $P$. falciparum, ultrasensitive molecular diagnostics that target multi-copy genes will be relevant to assess the true burden of non-falciparum malaria infections.

Despite recent reports of $P$. vivax detection in Duffy-negative individuals in SSA, including southwestern Nigeria, ${ }^{51}$ no $P$. vivax infection was detected. The prevalence of $P$. vivax in Duffynegative individuals could therefore be specific to some susceptible populations driven by yet undetermined biological and environmental factors.

In conclusion, the prevalence rates of non-falciparum species reported here indicate that approaches for routine diagnosis in endemic settings should be encouraged to ascertain the true burden of these species as well as for appropriate interventions toward pan-species malaria elimination. Studies to determine the prevalence and dynamics of non-falciparum species as well as associated risk factors in endemic populations need to be expanded across sSA.

Received June 4, 2020. Accepted for publication August 10, 2020.

Published online October 26, 2020.

Note: Supplemental figures appear at www.ajtmh.org.

Acknowledgments: We would like to thank Ernest Oriero for designing and adapting all maps used, Bekai Njie for the primary microscopy reads, and Sola Ajibaye for helping out with logistics in the Lagos site and the Statistics Department of the Medical Research Council Unit, the Gambia, at the LSHTM for support with the data analysis.

Financial support: This work was supported through the DELTAS Africa initiative (DELGEME Grant 107740/Z/15/Z). The DELTAS Africa initiative is an independent funding scheme of the African Academy of Sciences (AAS)'s Alliance for Accelerating Excellence in Science in Africa (AESA) and supported by the New Partnership for Africa's Development Planning and Coordinating Agency (NEPAD) with funding from the Wellcome Trust (DELGEME Grant 107740/Z/15/Z) and the UK government.

Authors' addresses: Eniyou C. Oriero, Umberto D'Alessandro, and Alfred Amambua-Ngwa, Medical Research Council Unit The Gambia at LSHTM, Banjul, The Gambia, E-mails: eoriero@mrc.gm, udalessandro@ mrc.gm, and angwa@mrc.gm. Adeola Y. Olukosi, Nigerian Institute of
Medical Research (NIMR), Lagos, Nigeria, E-mail: yaolukosi@yahoo. co.uk. Olabisi A. Oduwole and Martin M. Meremikwu, Calabar Institute of Tropical Disease Research and Prevention, University of Calabar Teaching Hospital, Calabar, Nigeria, E-mails: olabisioduwole $@$ gmail.com and mmeremiku@yahoo.co.uk. Abdoulaye Djimde, Department of Epidemiology of Parasitic Diseases, Malaria Research and Training Center, University of Science, Techniques and Technology of Bamako, Bamako, Mali, E-mail: adjimde@icermali.org.

This is an open-access article distributed under the terms of the Creative Commons Attribution (CC-BY) License, which permits unrestricted use, distribution, and reproduction in any medium, provided the original author and source are credited.

\section{REFERENCES}

1. WHO, 2019. World Malaria Report 2019. Geneva, Switzerland: World Health Organization. Available at: https://www.who.int/ publications-detail/world-malaria-report-2019. Accessed April 29, 2020.

2. Rabinovich RN et al., 2017. An updated research agenda for malaria elimination and eradication. PLoS Med 14: e1002456.

3. Mueller I, Zimmerman PA, Reeder JC, 2007. Plasmodium malariae and Plasmodium ovale - the 'bashful' malaria parasites. Trends Parasitol 23: 278-283.

4. Collins WE, Jeffery GM, 2007. Plasmodium malariae: parasite and disease. Clin Microbiol Rev 20: 579-592.

5. Langford S, Douglas NM, Lampah DA, Simpson JA, Kenangalem E, Sugiarto P, Anstey NM, Poespoprodjo JR, Price RN, 2015. Plasmodium malariae infection associated with a high burden of anemia: a hospital-based surveillance study. PLoS Negl Trop Dis 9: e0004195.

6. Abdurrahman MB, Aikhionbare HA, Babaoye FA, Sathiakumar N, Narayana PT, 1990. Clinicopathological features of childhood nephrotic syndrome in northern Nigeria. Q J Med 75: 563-576.

7. Hedelius R, Fletcher JJ, Glass WF, Susanti Al, Maguire JD, 2011. Nephrotic syndrome and unrecognized Plasmodium malariae infection in a US navy sailor 14 years after departing Nigeria. J Travel Med 18: 288-291.

8. Greenwood BM, Bradley AK, Greenwood AM, Byass P, Jammeh K, Marsh K, Tulloch S, Oldfield FS, Hayes R, 1987. Mortality and morbidity from malaria among children in a rural area of the Gambia, West Africa. Trans R Soc Trop Med Hyg 81: 478-486.

9. WHO, 2009. Malaria Rapid Diagnostic Test Performance - Results of WHO Product Testing of Malaria RDTs: Round 1 (2008). Geneva, Switzerland: World Health Organization Press.

10. Yman V et al., 2019. Persistent transmission of Plasmodium malariae and Plasmodium ovale species in an area of declining Plasmodium falciparum transmission in eastern Tanzania. PLoS Negl Trop Dis 13: e0007414.

11. Cooper DJ et al., 2019. Plasmodium knowlesi malaria in Sabah, Malaysia, 2015-2017: ongoing increase in incidence despite near-elimination of the human-only Plasmodium species. Clin Infect Dis 60: 361-367.

12. Federal Republic of Nigeria, 2016. The Federal Republic of Nigeria Nigeria Malaria Indicator Survey 2015 Final Report. Abuja, Nigeria: National Malaria Elimination Programme Federal Ministry of Health Federal Republic of Nigeria Abuja, Nigeria National Population Commission Federal Republic of Nigeria Abuja. Available at: www.DHSprogram.com. Accessed July 27, 2019.

13. May J, Mockenhaupt FP, Ademowo OG, Falusi AG, Olumese PE, Bienzle U, Meyer CG, 1999. High rate of mixed and subpatent malarial infections in southwest Nigeria. Am J Trop Med Hyg 61: 339-343.

14. Lagos, 2019. Wikipedia. Available at: https://en.wikipedia.org/w/ index. php?title=Lagos\&oldid=908437179. Accessed July 29, 2019.

15. Calabar, 2019. Wikipedia. Available at: https://en.wikipedia.org/ w/index.php?title $=$ Calabar\&oldid $=905748184$. Accessed July 29, 2019.

16. WorldWeatherOnline.com, 2019. Calabar Monthly Climate Averages. Available at: https://www.worldweatheronline.com/calabarweather/cross-river/ng.aspx. Accessed June 21, 2019. 
17. WHO, 2016. Malaria Parasite Counting: Malaria Microscopy Standard Operating Procedure - MM-SOP-09. Available at: http://www.wpro.who.int/mvp/lab_quality/2096_oms_gmp_sop_ 09_rev1.pdf. Accessed June 21, 2019.

18. Daniels RF et al., 2017. Evidence of non-Plasmodium falciparum malaria infection in Kédougou, Sénégal. Malar J 16: 9.

19. Hofmann N, Mwingira F, Shekalaghe S, Robinson LJ, Mueller I, Felger I, 2015. Ultra-sensitive detection of Plasmodium falciparum by amplification of multi-copy subtelomeric targets. PLoS Med 12: e1001788.

20. Yao B, Zhang L, Liang S, Zhang C, 2012. SVMTriP: a method to predict antigenic epitopes using support vector machine to integrate tri-peptide similarity and propensity. PLoS One 7: e45152.

21. Plasmodium Genomics Resource, 2018. PlasmoDB: The Plasmodium Genomics Resource. Available at: https://plasmodb.org/ plasmo/. Accessed March 18, 2020.

22. The Immune Epitope Database (IEDB), 2018. IEDB.org: Free Epitope Database and Prediction Resource. Available at: http:// www.iedb.org. Accessed March 18, 2020.

23. Corran PH et al., 2008. Dried blood spots as a source of antimalarial antibodies for epidemiological studies. Malar J 7: 195.

24. Takem EN et al., 2013. Detecting foci of malaria transmission with school surveys: a pilot study in the Gambia. PLoS One 8: e67108.

25. Fang R, Wey A, Bobbili NK, Leke RFG, Taylor DW, Chen JJ, 2017. An analytical approach to reduce between-plate variation in multiplex assays that measure antibodies to Plasmodium falciparum antigens. Malar J 16: 287.

26. Damen JG, Barnabas O, Damulak D, Ntuhun BD, Lugos MD, Nyary B, 2015. Malaria parasitemia in apparently healthy blood donors in north-central Nigeria. Lab Med 46: 42-46.

27. Olawumi HO, Fadeyi A, Babatunde SK, Akanbi AA, Babatunde AS, Sani MA, Aderibigbe SA, 2015. Malaria parasitaemia among blood donors in llorin, Nigeria. Afr J Infect Dis 9: 10-13.

28. Ugah UI, Alo MN, Owolabi JO, Okata-Nwali OD, Ekejindu IM, Ibeh $\mathrm{N}$, Elom MO, 2017. Evaluation of the utility value of three diagnostic methods in the detection of malaria parasites in endemic area. Malar J 16: 189.

29. Oboh MA, Badiane AS, Ntadom G, Ndiaye YD, Diongue K, Diallo MA, Ndiaye D, 2018. Molecular identification of Plasmodium species responsible for malaria reveals Plasmodium vivax isolates in Duffy negative individuals from southwestern Nigeria. Malar J 17: 439.

30. WHO, 2018. World Malaria Report 2018. Geneva, Switzerland: World Health Organization. Available at: http://apps.who.int/ iris/bitstream/handle/10665/275867/9789241565653-eng.pdf? $\mathrm{ua}=1$. Accessed December 19, 2018.

31. Mueller I, Widmer S, Michel D, Maraga S, McNamara DT, Kiniboro B, Sie A, Smith TA, Zimmerman PA, 2009. High sensitivity detection of Plasmodium species reveals positive correlations between infections of different species, shifts in age distribution and reduced local variation in Papua New Guinea. Malar J 8: 41.

32. Pemberton-Ross P, Smith TA, Hodel EM, Kay K, Penny MA, 2015. Age-shifting in malaria incidence as a result of induced immunological deficit: a simulation study. Malar J 14: 287.

33. Griffin JT, Ferguson NM, Ghani AC, 2014. Estimates of the changing age-burden of Plasmodium falciparum malaria disease in sub-Saharan Africa. Nat Commun 5: 3136.

34. Richie TL, 1988. Interactions between malaria parasites infecting the same vertebrate host. Parasitology 96: 607-639.

35. Bruce-Chwatt LJ, 1981. The Garki project. Research on the epidemiology and control of malaria in the Sudan savanna of West Africa. Trans R Soc Trop Med Hyg 75: 190-191.
36. Boudin C, Robert V, Verhave JP, Carnevale P, Ambroise-Thomas $\mathrm{P}, 1961$. Plasmodium falciparum and $P$. malariae epidemiology in a West African village. Bull World Health Organ 69: 199-205.

37. Rutledge GG et al., 2017. Plasmodium malariae and $P$. ovale genomes provide insights into malaria parasite evolution. Nature 542: 101-104.

38. Duez J, Holleran JP, Ndour PA, Pionneau C, Diakité S, Roussel C, Dussiot M, Amireault P, Avery VM, Buffet PA, 2015. Mechanical clearance of red blood cells by the human spleen: potential therapeutic applications of a biomimetic RBC filtration method. Transfus Clin Biol 22: 151-157.

39. Chotivanich K, Udomsangpetch R, McGready R, Proux S, Newton P, Pukrittayakamee S, Looareesuwan S, White NJ, 2002. Central role of the spleen in malaria parasite clearance. $J$ Infect Dis 185: 1538-1541.

40. Betson M, Sousa-Figueiredo JC, Atuhaire A, Arinaitwe M, Adriko M, Mwesigwa G, Nabonge J, Kabatereine NB, Sutherland CJ, Stothard JR, 2014. Detection of persistent Plasmodium spp. infections in Ugandan children after artemether-lumefantrine treatment. Parasitology 141: 1880-1890.

41. Dinko B, Oguike MC, Larbi JA, Bousema T, Sutherland CJ, 2013. Persistent detection of Plasmodium falciparum, P. malariae, $P$. ovale curtisi and $P$. ovale wallikeri after ACT treatment of asymptomatic Ghanaian school-children. Int J Parasitol Drugs Drug Resist 3: 45-50.

42. Rutledge GG et al., 2017. Genomic characterization of recrudescent Plasmodium malariae after treatment with artemether/lumefantrine. Emerg Infect Dis 23: 1300-1307.

43. Surendra $\mathrm{H}$ et al., 2019. Analysis of serological data to investigate heterogeneity of malaria transmission: a community-based cross-sectional study in an area conducting elimination in Indonesia. Malar J 8: 227.

44. Dewasurendra RL, Dias JN, Sepulveda N, Gunawardena GSA, Chandrasekharan N, Drakeley C, Karunaweera ND, 2017. Effectiveness of a serological tool to predict malaria transmission intensity in an elimination setting. BMC Infect Dis 17: 49.

45. Cook J et al., 2017. Sero-epidemiological evaluation of changes in Plasmodium falciparum and Plasmodium vivax transmission patterns over the rainy season in Cambodia. Malar J 11: 86.

46. Spencer HC, Collins WE, Chin W, Skinner JC, 1979. The enzymelinked immunosorbent assay (ELISA) for malaria. I. The use of in vitro-cultured Plasmodium falciparum as antigen. Am J Trop Med Hyg 28: 927-932.

47. Akpan GE, Adepoju KA, Oladosu OR, Adelabu SA, 2018. Dominant malaria vector species in Nigeria: modelling potential distribution of Anopheles gambiae sensu lato and its siblings with MaxEnt. PLOS ONE 13: e0204233.

48. Tatem AJ, Gething PW, Smith DL, Hay SI, 2013. Urbanization and the global malaria recession. Malar $J$ 12: 133.

49. Mehlotra RK, Howes RE, Cramer EY, Tedrow RE, Rakotomanga TA, Ramboarina S, Ratsimbasoa AC, Zimmerman PA, 2019. Plasmodium falciparum parasitemia and band sensitivity of the SD bioline malaria Ag P.f/Pan rapid diagnostic test in Madagascar. Am J Trop Med Hyg 100: 1196-1201.

50. Woodford $\mathrm{J}$ et al., 2019. An experimental human blood stage model for studying Plasmodium malariae infection. J Infect Dis 221: 948-955.

51. Twohig KA, Pfeffer DA, Baird JK, Price RN, Zimmerman PA, Hay SI, Gething PW, Battle KE, Howes RE, 2019. Growing evidence of Plasmodium vivax across malaria-endemic Africa. PLoS Negl Trop Dis 13: e0007140. 